\title{
Cell research with physically modified microfluidic channels: A review
}

\author{
Sun Min Kim, ${ }^{a}$ Sung Hoon Lee ${ }^{b}$ and Kahp Yang Suh*b \\ Received 16th January 2008, Accepted 17th April 2008 \\ First published as an Advance Article on the web 15th May 2008 \\ DOI: $10.1039 / \mathbf{b 8 0 0 8 3 5 c}$
}

An overview of the use of physically modified microfluidic channels towards cell research is presented. The physical modification can be realized either by combining embedded physical micro/nanostructures or a topographically patterned substrate at the micro- or nanoscale inside a channel. After a brief description of the background and the importance of the physically modified microfluidic system, various fabrication methods are described based on the materials and geometries of physical structures and channels. Of many operational principles for microfluidics (electrical, magnetic, optical, mechanical, and so on), this review primarily focuses on mechanical operation principles aided by structural modification of the channels. The mechanical forces are classified into (i) hydrodynamic, (ii) gravitational, (iii) capillary, (iv) wetting, and (v) adhesion forces. Throughout this review, we will specify examples where necessary and provide trends and future directions in the field.

${ }^{a}$ Department of Mechanical Engineering, Inha University, 253 Young Hyun-dong, Nam-gu, Incheon, 402-751, Republic of Korea

${ }^{b}$ School of Mechanical and Aerospace engineering and the Institute of Bioengineering, Seoul National University, San 56-1, Shinlim-dong, Kwanak-gu, Seoul, 151-742, Republic of Korea.

E-mail: sky4u@snu.ac.kr; Fax: +822-883-0179; Tel: +822-880-9103

\section{Introduction}

Over the past two decades lab-on-a-chip devices have made significant developments for analytical processes of biological and chemical samples in a single microfluidic device. ${ }^{1-5}$ Recently, the analysis of even more complex biological systems such as living cells with the use of microfluidic systems has attracted significant attention. Thus, microfluidic systems have started

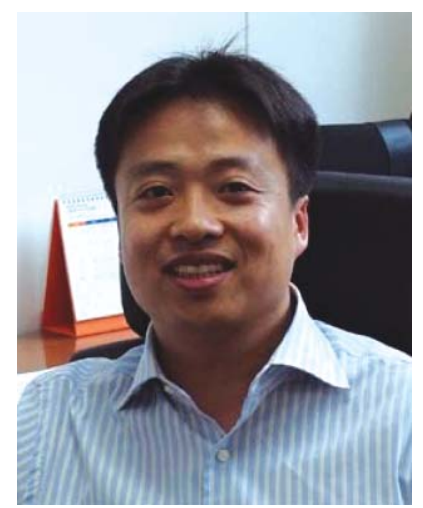

Sun Min Kim
Prof. Sun Min Kim received his BS (1997) and MS (1999) degrees in mechanical engineering from the Seoul $\mathrm{Na}$ tional University, Korea, and an MS degree in biomedical engineering and his $P h D$ degree in mechanical engineering from the University of Michigan, Ann Arbor in 2005 and 2006, respectively. In 2007, he joined the faculty of the Department of Mechanical Engineering, Inha University, following post-doctoral work at the Brigham and Women's Hospital, Harvard Medical School, MA, where he worked on the development of a cardiac cell based biosensor for toxin detection in water resources. He is interested in the fundamental understanding and development of micro/nanofluidic systems for biochemical sample analysis, cell-based biosensor, and cell analysis. He is currently working on the development of microfluidic systems for ultra-high sensitive immunoassay of clinical samples using electrokinetics.

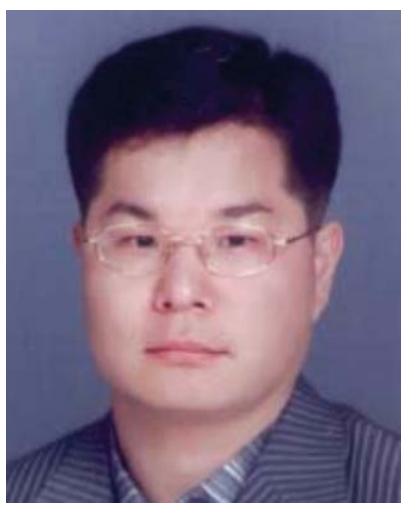

Kahp Yang Suh
Prof. Kahp-Yang Suh was born in Seoul, South Korea in 1972. After obtaining his PhD degree from the School of Chemical and Biological Engineering at Seoul National University (SNU) in 2002, he moved to MIT for postdoctoral research with Robert Langer, working on the merger of micro/nanotechnologies with tissue engineering and lab on a chip. He began his independent career in 2004 as an Assistant Professor in the Mechanical and Aerospace Engineering at SNU. He wrote 88 papers in peer-reviewed journals and 10 US or domestic patents. He won a number of awards, including the TR100 Young Innovator Award (2004) from MIT Technology Review, Best Graduate Student Award from the Brain Korea (BK) 21 Program of the Korean Government (2005) and the Young Professor Award from the College of Engineering at SNU (2007). His current research focuses on two areas: One is the integration of polymeric micro/nanostructures with microfluidic platforms for separation and enrichment of cancer cells and for single cell studies to elucidate signaling pathways of cellular expression. The other is mimicking functional nanostructures found in nature such as lotus leaf and gecko foot hairs using various unconventional lithographic methods. 
to play an increasingly important role in discoveries in cell biology, neurobiology, pharmacology, and tissue engineering. ${ }^{6-9}$ The major advantages of microfabricated systems for cell study are the ability to design cellular microenvironments, precisely control fluid flows, and to reduce the time and cost of cell culture experimentations. ${ }^{10,11}$

Traditionally, the role of a physically patterned microfluidic device (a microfluidic device containing a patterned surface topography or embedded structural components) is well represented by enhanced mixing and dispersion problems. ${ }^{12,13}$ To decrease the mixing length and mixing time, transverse flows must be generated, for which two general strategies are available, i.e., passive and active methods. ${ }^{14,15}$ These methods have usually benefited from incorporating physical microstructures or a patterned substrate inside a channel. In response to increasing demands for complex and improved structural functionalities of microfluidic systems, the physically modified microfluidic systems have been applied to cell studies. In particular, these systems are useful in various ways for performing unit operations with cells when suitably combined with mechanical operation principles. Here, these mechanical principles are categorized into (i) hydrodynamic, (ii) gravitational, (iii) capillary, (iv) wetting, and (v) adhesion forces.

Some exemplary works are in order. First, the physical structures can manipulate cells in a microchannel by changing hydrodynamic flow profiles. Constrictions included in a microfluidic channel can work as filters for sized-based cell separation and enrichment by controlling hydrodynamic forces. This cell separation technique could be most useful for cells for which markers are yet to be found. The design of these structures often requires unusual geometries and the interplay of multiple physical effects such as pressure gradients, electrokinetics, gravity, and capillarity for flow profile controls. Second, the physically modified channels can be used to capture cells in shear-free locations by exploiting capillary force or gravitational sedimentation to prevent the modification of cell behavior by shear stress. Third, the physical structures in a microchannel can change wetting properties of a fluid and guide adhesion, organization, and functionality of cells. This engineered cell culture substrate can induce changes in cell morphology and mobility, which can act as a microfluidic platform for elucidating cell signaling pathways or emerging issues in tissue engineering.

Although several review articles regarding cell analysis using microfluidic systems are available in the literature, ${ }^{6,7,16-19}$ we mainly focus on the microfluidic systems that are combined with physical micro/nanostructures or a topographically patterned substrate for cell research in this review. These physical structures give rise to a notable change in mechanical forces in various ways, allowing for a low-expertise route to manipulation of cells inside a channel under dynamic or static flow conditions.

\section{Fabrication methods}

A traditional method of creating microfluidic systems containing structural components is to bond two layers with alignment: a substrate layer constructed with micro/nanostructures on the surface (a micro- or nanostructured layer) and a channel layer with the impression of microchannels. In addition, various micro/nanofabrication techniques can be used for manufacturing physically modified microfluidic systems. In general, fabrication methods should be chosen depending on the materials and geometries that are used for physical structures and channels. Table 1 summarizes a broad range of fabrication methods based on the materials used in the method. Here, five fabrication methods for fabricating channel and structural components are conceivable. First, conventional MEMS processes (photolithography, electroplating, deposition, and etching) are used to create structures on Si or glass substrates and then a soft lithography or etching process forms a microchannel with polydimethylsiloxane (PDMS) or glass. Then, a microstructured layer is bonded with a microchannel layer to form a microfluidic channel. ${ }^{20-25}$ Second, multi-layered PDMS based microfluidic systems are fabricated with one or multiple uses of the soft lithographic process. One approach here is that two different PDMS layers, one with a channel and the other with micro/nanostructures, are separately fabricated and bonded with alignment. ${ }^{26-30}$ The other approach is that a channel layer containing physical network structures is fabricated on a PDMS layer replicated from a multi-layered master mold and this PDMS layer is bonded with a flat PDMS or glass substrate. ${ }^{31-33}$ All the steps are based on well-established replica moulding and thus a complicated two-dimensional or three-dimensional channel network can be generated in a relatively simple manner. Third, transparent microfluidic channels are fabricated with PDMS or glass by soft lithography or etching. Then, a photo-curable hydrogel solution fills the channel and UV light patterns or creates microstructures inside microchannels as designed. ${ }^{34-36}$ Fourth, the soft lithography technique creates engraved microstructures with polymer materials such as PDMS and UV-curable hydrogels/resins. The PDMS based microchannel layer is bonded with a polymer layer to obtain a microfluidic channel with physical structures. ${ }^{37-41}$ Fifth, micro/nanoscale structures are formed on a polymer layer or Si substrate by nanoimprinting, soft lithography, or reactive ion etching following a suitable lithographic process. Subsequently, a microchannel layer of PDMS or glass is bonded to form a microfluidic channel. ${ }^{42-44}$

Another classification is conceivable based on the geometry of structures on the bottom of channels, for which three different scenarios are illustrated in Fig. 1. First, the height of structures included in the channel $(h)$ is comparable to that of the channel height $(H)$, thereby modifying hydrodynamic flow profiles in a totally different fashion $(H-h<H)$. Many cell sorting, trapping and enrichment methods are based on this strategy. Second, $h$ is negative in the case of engraved microstructures $(H-h>H)$, which are particularly useful for capturing cells in pre-defined locations within a channel, without altering flow profiles extensively. Cell docking under static conditions (sedimentation) or surface-tension driven flows are typical examples. Finally, $h$ is much lower than $H(h<<H)$, resulting in the modification of the substrate topography without altering flow characteristics except for wetting and friction resistance between a fluid and a channel wall.

In addition to the above-mentioned techniques, many other fabrication techniques including laser machining, micromechanical machining, and ink jet printing, are also applicable to create structure-embedded microfluidic systems. However, it should be noted that in many instances, more than one fabrication process can be implemented to achieve the final 
Table 1 Fabrication methods for physically modified microfluidic channels based on materials

\begin{tabular}{|c|c|c|c|c|c|c|}
\hline \multicolumn{2}{|l|}{ Materials } & \multicolumn{2}{|l|}{ Fabrication methods } & \multirow[b]{2}{*}{ Scheme (see Fig. 1) } & \multirow[b]{2}{*}{ Applications } & \multirow[b]{2}{*}{ References } \\
\hline Structures & Micro-channel & Structures & Microchannel & & & \\
\hline $\mathrm{Si}$, glass & PDMS, glass & $\begin{array}{l}\text { Conventional MEMS } \\
\text { processes } \\
\text { (photolithography, } \\
\text { deposition, and } \\
\text { etching) }\end{array}$ & $\begin{array}{l}\text { Replica moulding, } \\
\text { etching }\end{array}$ & Route 1 & $\begin{array}{l}\text { Cell trapping, } \\
\text { separation and } \\
\text { enrichment. - Single } \\
\text { cell manipulation }\end{array}$ & $20-24,47$ \\
\hline PDMS & PDMS & Replica moulding & Replica moulding & Route 1,2 & $\begin{array}{l}\text { Cell separation, } \\
\text { culture, and } \\
\text { enrichment. - Single } \\
\text { cell manipulation }\end{array}$ & $\begin{array}{l}25,27- \\
30,32,33,45,46\end{array}$ \\
\hline Hydrogel & PDMS, Glass & Photo-polymerization & $\begin{array}{l}\text { Replica moulding, } \\
\text { etching }\end{array}$ & Route 1 & $\begin{array}{l}\text { Cell manipulation, } \\
\text { culture, and trapping }\end{array}$ & $34-36$ \\
\hline $\begin{array}{l}\text { PDMS, hydrogel, } \\
\text { PMMA }\end{array}$ & PDMS & Soft lithography & Replica moulding & Route 2,3 & $\begin{array}{l}\text { Cell docking, and } \\
\text { culture }\end{array}$ & $37,38,40,41$ \\
\hline $\begin{array}{l}\text { Si, hydrogel, } \\
\text { PMMA }\end{array}$ & PDMS, glass & $\begin{array}{l}\text { Nanofabrication } \\
\text { processes } \\
\text { (nanoimprinting, soft } \\
\text { lithography, and } \\
\text { reactive ion etching) }\end{array}$ & $\begin{array}{l}\text { Replica moulding, } \\
\text { etching }\end{array}$ & Route 3 & $\begin{array}{l}\text { Contact } \\
\text { guidance-control of } \\
\text { wettability }\end{array}$ & $42-44$ \\
\hline
\end{tabular}

\section{Micro/Nanostructure}

fabrication

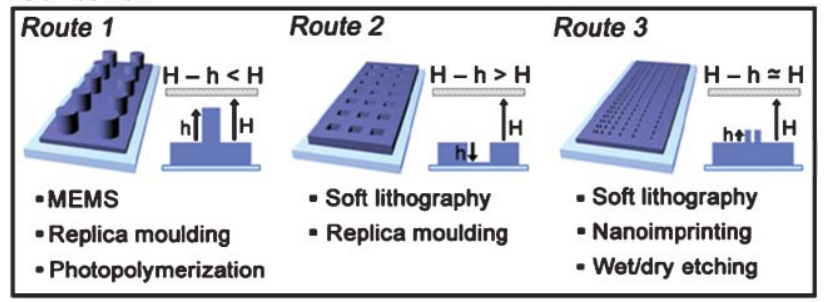

Microchannel

fabrication \& bonding

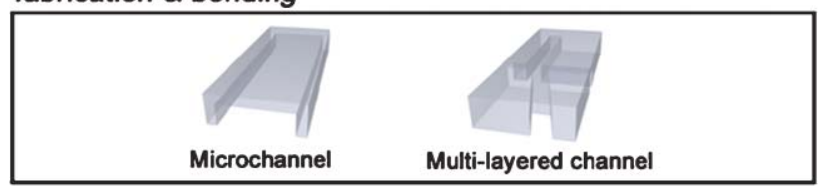

Physically modified

microfluidic devices

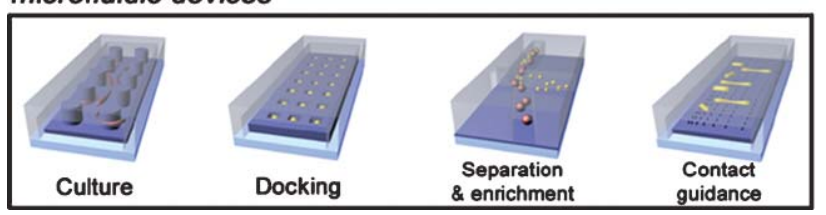

Fig. 1 Fabrication schemes of physically modified microfluidic channels based on relative size of micro- and nanostructures compared to channel dimension.

production of a device. Also, the selection of materials of these microfluidic systems should be carefully considered at the design and the fabrication steps for cell viability and processability.

\section{Mechanical principles}

A number of mechanical principles should come into play when combined with structure-embedded microfluidic channels. Of these, five different mechanical forces are considered here: hydrodynamic, gravitational, capillary, wetting, and adhesion forces. A common characteristic among these forces is a passive operation under dynamic or static flow conditions without involving other external stimuli such as electrical, magnetic, and optical manipulation. Thus, control over these physical forces can be easily implemented by exploiting material properties of cells or fluids or by changing channel geometry.

\subsection{Hydrodynamic force}

Fluid flows in microchannels have very low Reynolds number (generally $<0.1$ ) due to small channel diameter, so the flows are predominantly laminar. Such laminar flows can precisely transport and manipulate cells throughout microfluidic channels with appropriate microfluidic design and fluid flow control. ${ }^{8}$ In this regard, controlled transport, immobilization, and manipulation of cells are important functions to be incorporated into a microfluidic device in order to carry out on-chip biochemical and cell biological experiments. ${ }^{7}$

Under hydrodynamic conditions, velocity profiles in simple two-dimensional geometries are parabolic in pressure-driven flows, nearly constant in electro-osmotic flows, or a superposition of both in the presence of combined flows. In a typical pressure-driven situation, flows can be described by Stokes equations for incompressible motions with no-slip boundary conditions, which is given by

$$
F_{\mathrm{h}}=6 \pi \eta R u
$$

where $F_{\mathrm{h}}$ is the drag force exerted on the cell surface, $\eta$ is the viscosity of a fluid, $R$ is the radius of cells (assuming a round shape), and $u$ is the velocity of a fluid. Viscosity is constant for a given fluid and so velocity or cell size can be controlled to manipulate cell motions in a channel. Based on this principle, Yamada et al. presented microfluidic systems of hydrodynamic filtration for continuous and size-dependent cell separation using a channel network including branch channels or bifurcations. ${ }^{45,46}$ By introducing cell suspension into a microchannel with multiple side-branch channels, cells could be accurately and passively separated according to size 
difference. The operation of this system is based on a difference in hydrodynamic force exerted on the cells with different size along with proper fluid control.

Inclusion of physical obstacles in a microfluidic channel is an alternative way of changing hydrodynamic profiles by modifying local velocities of cells. Davis et al. reported a microfluidic device employing "deterministic lateral displacement" around obstacles ${ }^{47}$ to separate blood cells and other blood components by their hydrodynamic size, independent of their mass. ${ }^{48}$ This device has an array of microposts, in which posts are located in consistent gap between the posts in each row. In addition to this work, various strategies have been introduced based on channel modification by physical structures. For example, Choi et al. proposed a new hydrophoretic method for continuous blood cell separation using a microfluidic device having physical obstacles. ${ }^{30}$ The physical obstacles were composed of slanted obstacles for particle focusing and filtration obstacles for separation of small and large particles. Di Carlo et al. reported a microfluidic device composed of arrays of physical U-shaped hydrodynamic trapping structures for dynamic single cell culture. ${ }^{25}$ Each trapping structure is biased to capture only a single HeLa cell without any surface modification. Ryley and Pereira-Smith developed a microfluidic device containing $\mu \mathrm{m}-$ sized structures (yeast jails) to physically trap individual yeast for measuring single-cell gene expression over time. ${ }^{29}$ Valero et al. presented the design and the fabrication of a microfluidic cell trap device for analysis of apoptotic cell death dynamics. ${ }^{24}$ The silicon-glass microfluidic device enabled the immobilization of cells and real-time monitoring of the apoptotic process. Yang et al. developed a microfluidic device which has a dam structure to capture cells for on-chip monitoring of cellular reactions. ${ }^{20}$ In this device, a dam structure in parallel to the fluid flow was constructed for docking and alignment of biological cells, which allows the fragile cells to move in the microfluidic channels and to be immobilized with controllable numbers in desired locations. Li et al. fabricated multi-height sandbag structures to immobilize cells using single step photolithography. ${ }^{21}$ A microfluidic device including sandbag structures was easily produced with deformable PDMS substrates. Fig. 2 shows two schemes of cell manipulation based on hydrodynamic force and one example of such manipulation to capture cells in a continuous flow stream.
For details, several review articles are available on the use of hydrodynamic flows for cell manipulation..$^{6,7,18,49}$

Microfluidic devices using hydrodynamic flows exhibit numerous advantages, such as non-marker labeling, short detection time, and high reproducibility based on simple and robust experimental procedures. Hence this approach can be used to isolate rare cells that do not express known markers. Furthermore, the size-based approach is relatively less invasive because it does not require any chemical or biological interactions between the cells and the device. However, several potential shortcomings are envisioned. First, direct applications to cellbased assay are limited by poor selectivity without biological labeling. The poor selectivity could be enhanced by amplifying physical differences using controlled micro/nanoenvironments in microfluidic channels. Second, a mechanical stress is exerted on a captured or flowing cell, which could give rise to cell deformation and shear-induced changes in cell behavior. A careful consideration on the channel design and flow control is required to address this issue. Finally, many methods developed so far lack control over surface chemistry or topography for anchorage-dependent cells within the captured microstructures, which would limit widespread uses to most mammalian cells, in particular, for long-time cultures. The development of simple, yet robust techniques for fabricating microstructures within microchannels with precise control over surface properties is potentially of benefit.

\subsection{Gravitational force}

Gravitational force or sedimentation is familiar to most biologists and offers the simplest, low-expertise route to manipulating cells within a channel or without a channel. Frequently, it is necessary to capture cells within particular regions of a channel for high-throughput screening of single to multiple cells or optimization of cell-soluble signal interactions for biological research or tissue engineering. When combined with microfluidics, structural compartments enable immobilization of cells within pre-defined locations within microchannels. For this purpose, several methods have been introduced, which include laminar flow patterning, ${ }^{50}$ pre-patterning with adhesive ligands, ${ }^{51}$ and immobilization inside hydrogels. ${ }^{52}$ However, there
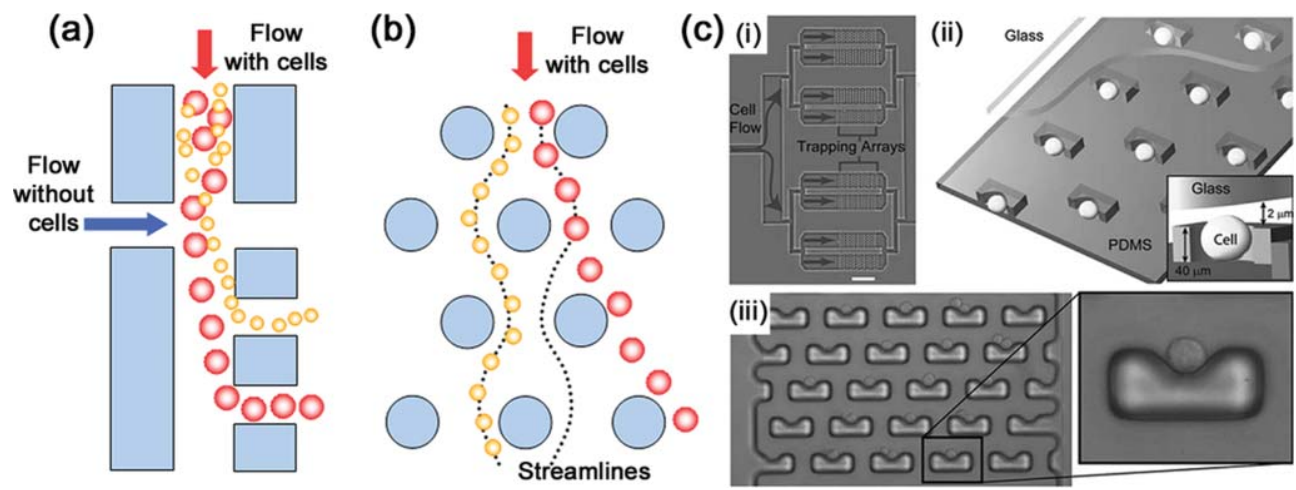

Fig. 2 Manipulating cell motion by hydrodynamic force. (a) A schematic diagram of continuous and size-dependent cell separation. (b) A schematic diagram of changing hydrodynamic flow profile by micro-obstacles. (c) An example of a cell trapping device using hydrodynamic force. (i) A photograph of the U-shaped hydrodynamic cell trapping array. (ii) A schematic view of the cell trapping mechanism. (iii) A high resolution brightfield micrograph of the array of trapped cells. Reprinted with permission from ref. 25. 
are potential limitations of these approaches. Laminar flow patterning can only pattern the limited shape of patterned regions, and hydrogel fabrication using UV radiation induces the exposure of cells to potentially toxic environments. Also, the direct patterning of cells on the pre-patterned substrate of the channel could give rise to shear driven modifications in cell behavior. To overcome these limitations, physically modified microfluidic systems have been developed to capture and localize cells by exploiting gravitational force. ${ }^{20,37-39,41,53-55}$

Khademhosseini et al. introduced a simple soft lithographic technique to fabricate PEG microstructures within microfluidic channels that can immobilize cells within specific locations. ${ }^{37}$ Microstructures of various shapes were used to capture cells despite shear flow in the channel. Using soft lithography and reversible sealing, an advanced microwell system was also fabricated for multiphenotype cell patterning within an array of reversibly sealed microfluidic channels. ${ }^{38}$ Rettig and Folch presented a method for high-throughput measurements of cellular response using soft-lithographically fabricated PDMS microwells. ${ }^{54}$ This technique can achieve the trapping of single cells with high efficiency in large arrays of microwells. Deutsch et al. fabricated a densely packed 2-D arrangement of hexagonal picolitre wells made of glass, in a honeycomb-like pattern for cell trapping. ${ }^{55}$ Love et al. introduced a soft lithography technique for microengraving using a dense array of replica moulded PDMS microwells. ${ }^{39}$ An array of microwells filled with cells was sealed with a pre-treated glass slide and the glass slide was detached from the array after incubation, allowing for rapid and high-throughput analysis of cells. Lee et al. fabricated bottleshaped, hollow polymeric microstructures inside a microfluidic channel for potential shear-protecting cell reservoirs. ${ }^{41}$ The hollow bottle-shaped structures were generated by exploiting partial capillary rise with a controlled amount of polymer solution. Fig. 3 shows a schematic diagram of capturing cells by sedimentation and one example of using micro-moulded PEG structures.

Although sedimentation provides a simple, low-expertise route to docking cells in engraved or hollow structures, its efficiency is relatively low with poor reproducibility. Cells are usually deposited non-uniformly over an area with many empty sites. This is because cell docking efficiency is determined by local dispersion of cell solution as well as flow conditions. An improved design or experimental set up implemented by hydrodynamics will further expand the use of gravitational force in cell manipulation.

\subsection{Capillary force}

As dimensions shrink to microscale, the relative importance of surface to volume forces increases. In the presence of a gasliquid or liquid-liquid interface, fluid or particle motion can be generated by controlling spatial variations of surface tension. According to the Young-Laplace equation, a capillary force arises when a liquid meets a solid wall, which is given by

$$
F_{\mathrm{c}}=\frac{2 \gamma}{r} \cos \theta
$$

where $\gamma$ is the surface tension of a fluid, $r$ is the radius of a channel (assuming a pipe geometry), and $\theta$ is the contact angle at the three phase contact line. The motion of a fluid or a particle, thus, can be generated by varying along the channel either wetting properties (contact angle and surface tension) or geometrical features (channel diameter). However, this principle usually has been applied to move a liquid droplet within a channel by using thermal gradients ${ }^{56}$ or variation of channel width without a power supply. ${ }^{57}$

Capillary interaction between colloidal particles has been extensively studied both experimentally ${ }^{58,59}$ and theoretically ${ }^{60}$ to produce formation of a two-dimensional lattice structure upon evaporation. Also, a capillarity or surface tension driven method has been introduced to fabricate a colloidal assembly with wellcontrolled sizes, shapes, and structures on surfaces patterned with two-dimensional arrays of relief structures. ${ }^{58}$ Recently, this strategy was directly applied to capture cells in pre-defined locations within a channel. Park et al. proposed a cell docking method aided by a receding meniscus formed upon natural evaporation of the medium solution. ${ }^{40} \mathrm{~A}$ schematic is shown in Fig. 4 along with an example of a captured array. Engraved microwells were fabricated either by soft lithographic capillary moulding of UV curable PUA resin onto glass substrate or direct replica moulding of PDMS. Here, cells were spontaneously captured in the center of microwells to minimize the lateral

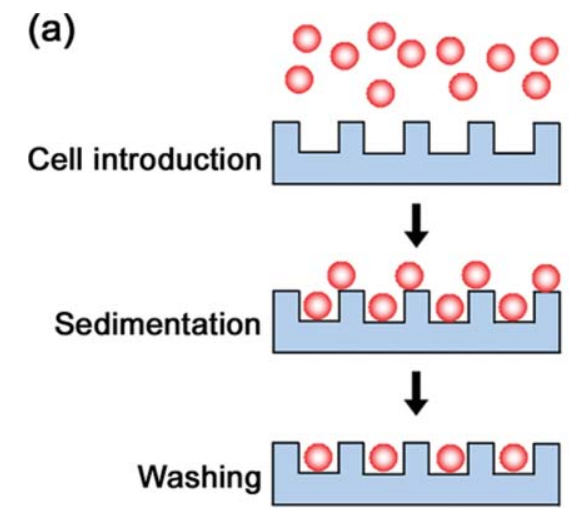

(b)
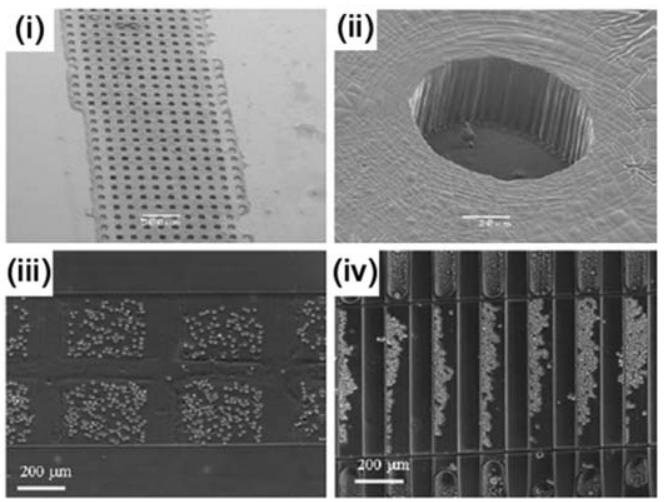

Fig. 3 Capturing and localizing cells by sedimentation. (a) A schematic diagram of trapping cells within engraved microstructures. (b) An example of the captured cell array. (i) A large-area SEM image of molded PEG microwells. (ii) A magnified view of an individual circular microwell. Docked cells within microstructures of various sizes and shape such as (iii) grids and (iv) perpendicular lanes. Reprinted with the permission from ref. 37. 
(a)

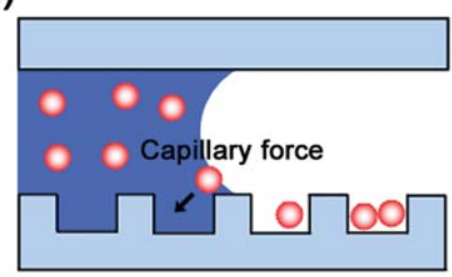

(b)

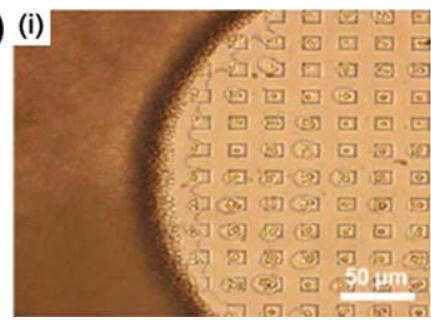

(ii)

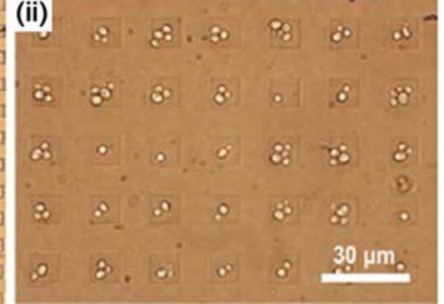

Fig. 4 Capturing and localizing cells by capillary force. (a) A schematic diagram of cell docking within engraved microwells by capillarity-driven flow. (b) (i) An example of the docked cell array using yeast cells by receding meniscus and (ii) a magnified brightfield image of captured yeast cells within $10 \mu \mathrm{m}$ microwells. Reprinted with permission from ref. 40 .

capillary force created at the bottom of the receding meniscus. Since capillary force is an effective way of flowing a liquid into a microfluidic channel, this cell docking scheme can be performed without additional accessories such as a syringe pump or electric power. Cell viability might be a concern because the cells are exposed to air for a period of time ( $<5 \mathrm{~min})$, which should be addressed prior to application to cell-based assay.

In addition to the cell docking strategy mentioned above, capillary force also plays an important role in cell manipulation in the form of cell-cell capillary interactions under dynamic or static flow conditions. It is known that two similar particles (or cells) floating on a liquid interface attract each other because the liquid meniscus deforms and the gravitational potential energy decreases when they approach each other. The origin of this force is the particle weight (later flotation forces). Furthermore, capillary attraction also occurs when the particles are partially immersed in a liquid layer on a substrate. The attraction is related to the wetting properties of the particle surface rather than gravity (later immersion forces). According to a previous study, ${ }^{60}$ the flotation and immersion forces exhibit similar dependence on the interparticle separation but different dependencies on the particle radius and the surface tension of the liquid, which are represented by

$$
F_{\mathrm{c}, \mathrm{f}} \propto \frac{R^{6}}{\gamma} f(L) \text { for flotation force }
$$

$$
F_{\mathrm{c}, \mathrm{i}} \propto \gamma R^{2} f(L) \text { for immersion force }
$$

where $F_{\mathrm{c}, \mathrm{f}}$ and $F_{\mathrm{c}, \mathrm{i}}$ are the flotation and immersion forces, respectively, $R$ is the radius of particles, and $f(L)$ is a function of the interparticle spacing, $L$. Therefore, the flotation force, which could affect floating cells at the front in a continuously flowing stream, increases when the interfacial tension decreases. This is readily understood in that at given particle weight the meniscus deformation is larger when $\gamma$ is lower. On the other hand, the immersion force, which affects captured cells in engraved structures in the above cell docking strategy, increases in proportion to $\gamma$. This is attributed to the fact that at a given capillary rise the meniscus surface energy is larger when $\gamma$ is higher. Consequently, the hydrodynamic force needs to overwhelm the lateral flotation force in order to manipulate a high-density cell solution at single cell level. In a similar manner, cell density needs to be smaller than a certain value to capture cells into engraved structures at single cell level.

\subsection{Wetting force}

The wettability of a solid surface is a very important physical property for cells that is determined by a combination of chemistry and physics; i.e., the chemical composition and the geometrical microstructures of the surface.$^{61-63}$ When a relatively large roughness is present on a solid surface, the wettability is governed by heterogeneous wetting (so called Cassie state) where air can be trapped in the spaces between structural features. This levitation enhances the hydrophobicity of the surface because the drop is then partially sitting on air, as can be found in a lotus leaf. ${ }^{64,65}$

Cassie and Baxter proposed an equation that describes the apparent contact angle on a composite surface that is composed of solid and air, which is given by ${ }^{66}$

$$
\cos \theta^{c}=f(\cos \theta+1)-1
$$

where $\theta^{\mathrm{c}}$ is the apparent contact angle on a composite surface determined by Cassie state, $\theta$ is the equilibrium contact angle on a flat surface, and $f$ is the area fraction of the solid. According to the above equation, $\theta^{c}$ increases with decreasing $f$ because more air is trapped in the roughness and then serves as a hydrophobic cushion reducing the contact area of a water droplet with the solid surface. When superhydrophobic surfaces are constructed inside a channel on one or both sides of substrates, a pressure drop is drastically decreased due to an increased slip length at the channel wall. A recent study demonstrated that superhydrophobic surfaces can be generated inside a microfluidic channel by forming high density arrays of tall and sharp nanoposts (so-called "nanoturfs") with a submicron pitch on the top and bottom substrates. ${ }^{43}$ Although this device has not been examined for controlling cell adhesion, initial cell attachment would be significantly reduced. However, a long-term exposure to a fluid flow could ultimately lead to homogeneous wetting of a fluid and subsequent cell spreading, for which a further study is required.

\subsection{Adhesion force}

Cells are inherently sensitive to local mesoscale, microscale, and nanoscale topographic and molecular patterns in extracellular matrix (ECM) environment. ${ }^{67-69}$ Integration of microfluidics and micro/nanofabrication methods, thus, can be employed to precisely control cell adhesion and growth on a topographically patterned substrate within a fluidic channel. Structures included inside a microchannel are useful in various ways. First, they can control cell adhesion and induce changes in morphology 
and motility of many different cell types without chemical stimuli, a phenomenon called contact guidance. ${ }^{70,71}$ The micro/nanotopography induces the physical stimulation, which affects the cell organizations and functionality. Also, controlled cell adhesion by micro/nanotopography with microfluidics can be used for detecting and separating cells of interest without labeling. ${ }^{44}$

After a pioneering work by Chen and coworkers, micro/nanoscale topographic features have been incorporated into the in vitro experimental platform to mimic various in vivo $3 \mathrm{D}$ ECM environments with structural and mechanical similarity by using advanced fabrication methods. ${ }^{72,73}$ To elaborate on the design of the biomaterials interface within a channel, Zaari et al. created substrates with variations in mechanical compliance by combining microfluidics and photopolymerization. ${ }^{74}$ In this integrated platform, a well-controlled gradient-compliance profile on the microscale was used to study cell migration guided by substrate rigidity (so called "durotaxis"). In additional to this chemical tuning by controlling the crosslinking density of hydrogels, one can control adhesion and growth of cells by using a topographically patterned substrate. ${ }^{75,76}$ For example, Martines et al. presented a microfluidic device having nanopits in a microchannel to investigate cell adhesion onto nanopits under dynamic conditions, by means of a shear flow. ${ }^{42}$ Dynamic cell adhesion was quantified and compared on flat and nanopitted polymethyl methacrylate (PMMA) substrates with cell suspension flow. Kwon et al. recently developed a label-free microfluidic method for separation and enrichment of human breast cancer cells using controlled cell adhesion as a physical marker. ${ }^{44}$ The nanostructured polymer surfaces $(400 \mathrm{~nm}$ pillars, $400 \mathrm{~nm}$ perpendicular or $400 \mathrm{~nm}$ parallel lines) were constructed on the bottom of PDMS microfluidic channels in three of four parallel channels using a UV-assisted capillary moulding technique to maximize the adhesion difference between human breast epithelial cells (MCF10A) and cancer cells (MCF7). A schematic of cell manipulation principle based on adhesion force is shown in Fig. 5 along with an example of a device with physically patterned channels.
The topographically patterned substrate within a microfluidic channel could not only open opportunities for understanding and tailoring cell adhesion and growth but also serve as a template for better tissue engineering and controlling cellular activities. In addition, the controlled adhesion using micro/nanostructures in a microfluidic device can be used as a label-free method for separation and enrichment of cancer cells from body fluid containing a mixed population of normal and cancer cells. For cell-based assays or tissue engineering, materials need to be biocompatible with good processability. Also, relatively smooth surfaces are required with dimensions relevant to biological samples such as cell, bacteria and yeast. Future studies would be directed towards construction of the in vivo like environments within a fluidic channel with advanced fabrication methods.

\section{Conclusions and future directions}

Microfluidic systems have been applied for controlled transportation, immobilization, and manipulation of biological molecules and cells, as well as separation, mixing, and dilution of chemical reagents, which enables the analysis of intracellular parameters and detection of cell metabolites. Typically, cell manipulation methods in microfluidic systems are based on magnetic, optical, electrical and mechanical principles. This review is devoted to present the application of microfluidic systems for cell research based on mechanical operation principles aided by structural modification of channels under dynamic or static flow conditions.

The physically modified microfluidic channels, when suitably combined with controlled fluid flow and mechanical operation principles, are beneficial to cell research. Since the size of microstructures can be tuned to various length scales compared to that of cells thanks to advanced micro/nanofabrication methods, the microstructures either can act as cell manipulating obstacles or elements for mimicking in vivo like microenvironments. Specifically, the physical structures in a microfluidic system can (i) separate cells based on size without labeling (a)

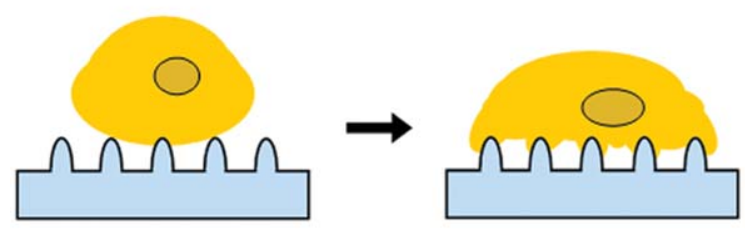

(b)

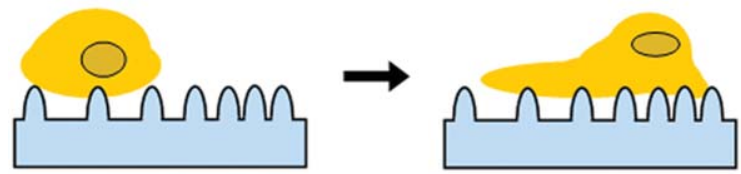

(c)

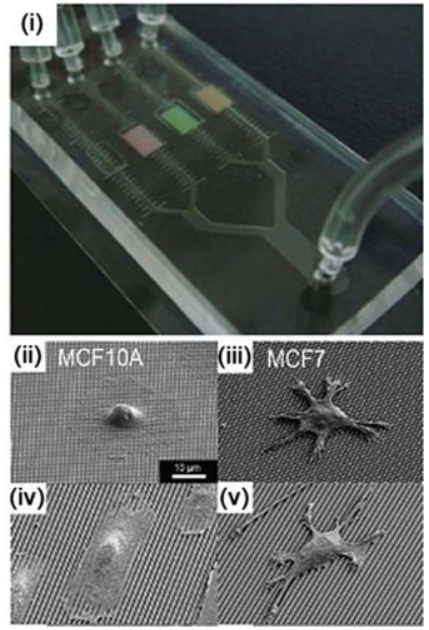

Fig. 5 Control of cell behavior by adhesion force. (a) A schematic diagram for adhesion of cells onto a topographically patterned substrate. (b) A schematic diagram for guiding cell migration by micro/nanotopography. (c) An example of a microfluidic device using cell adhesion as a physical marker. (i) A photograph of a microfluidic channel having four branch channels with a patterned substrate. SEM images of MCF10A cell on $400 \mathrm{~nm}$ pillars (ii) and $400 \mathrm{~nm}$ lines (iv), and MCF7 cells on $400 \mathrm{~nm}$ pillars (iii) and $400 \mathrm{~nm}$ lines (v). Reprinted with the permission from ref. 44. 
markers, (ii) manipulate cells without shear driven modification of cell behavior, and (iii) control cell adhesion and growth by controlling the surface properties of the cell substrates. Although not addressed in detail, other cell treatments and manipulations such as cell lysis, cell culture, and single cell manipulation also have been performed in microfluidic systems including physical structures. ${ }^{22,25,29,32,77,78}$

Recently, much attention has been paid to single cell culture and analysis. The single cell analysis provides useful information on the significant biochemical heterogeneity existing among cells of the same type. Aiming at this field, structure-embedding microfluidic systems are beneficial for arrayed culture of individual adherent cells and dynamic control of fluid perfusion with uniform environments for individual cells. If further manipulated and optimized, the physically modified microfluidic channels would provide a powerful tool for examining drug toxicity with physiologically relevant perfused dosages as well as for investigating cell signaling pathways and systems biology.

Even though physically modified microfluidic systems have several advantages in cell studies, several problems such as poor selectivity, time consumption for optimizing fluidic control, fabrication reliability, and user interface need to be addressed to further advance the application of the systems. Furthermore, the integration of other manipulation techniques based on chemical, electrical, magnetic and optical principles will generate a large variety of microfluidic systems for cell studies. The current efforts to development of smart functional materials and advanced micro/nanofabrication technologies will expedite use of physically modified microfluidic systems in various applications.

\section{Acknowledgements}

This work was financially supported by INHA UNIVERSITY Research Grant (INHA-36604) for Sun Min Kim. This work was also supported by the Korea Research Foundation Grant funded by the Korean Government (MOEHRD, Basic Research Promotion Fund) (KRF-1006-003-D00040).

\section{References}

1 A. Van Den Berg and T. S. J. Lammerink, in Microsystem Technology in Chemistry and Life Science, 1998, vol. 194, pp. 21-49.

2 A. J. Tudos, G. A. J. Besselink and R. B. M. Schasfoort, Lab Chip, 2001, 1, 83-95.

3 D. R. Reyes, D. Iossifidis, P. A. Auroux and A. Manz, Anal. Chem., 2002, 74, 2623-2636.

4 P. A. Auroux, D. Iossifidis, D. R. Reyes and A. Manz, Anal. Chem., 2002, 74, 2637-2652.

5 E. Verpoorte, Electrophoresis, 2002, 23, 677-712.

6 H. Andersson and A. Van Den Berg, Sens. Actuators, B, 2003, 92, 315-325.

7 C. Q. Yi, C. W. Li, S. L. Ji and M. S. Yang, Anal. Chim. Acta, 2006, 560, $1-23$.

8 J. El-Ali, P. K. Sorger and K. F. Jensen, Nature, 2006, 442, 403-411.

9 A. Khademhosseini, R. Langer, J. Borenstein and J. P. Vacanti, Proc. Natl. Acad. Sci. U. S. A., 2006, 103, 2480-2487.

10 T. H. Park and M. L. Shuler, Biotechnol. Prog., 2003, 19, 243-253.

11 G. M. Walker, H. C. Zeringue and D. J. Beebe, Lab Chip, 2004, 4, 91-97.

12 A. D. Stroock, S. K. W. Dertinger, A. Ajdari, I. Mezic, H. A. Stone and G. M. Whitesides, Science, 2002, 295, 647-651.

13 N. S. Lynn and D. S. Dandy, Lab Chip, 2007, 7, 580-587.
14 V. Hessel, H. Lowe and F. Schonfeld, Chem. Eng. Sci., 2005, 60, 2479-2501.

15 N. T. Nguyen and Z. G. Wu, J. Micromech. Microeng., 2005, 15, R1-R16.

16 S. K. Sia and G. M. Whitesides, Electrophoresis, 2003, 24, 3563-3576.

17 T. Vilkner, D. Janasek and A. Manz, Anal. Chem., 2004, 76, 33733385.

18 D. Huh, W. Gu, Y. Kamotani, J. B. Grotberg and S. Takayama, Physiol. Meas., 2005, 26, R73-R98.

19 M. Toner and D. Irimia, Annu. Rev. Biomed. Eng., 2005, 7, 77-103.

20 M. S. Yang, C. W. Li and J. Yang, Anal. Chem., 2002, 74, 3991-4001.

21 C. W. Li, C. N. Cheung, J. Yang, C. H. Tzang and M. S. Yang, Analyst, 2003, 128, 1137-1142.

22 D. Di Carlo, K. H. Jeong and L. P. Lee, Lab Chip, 2003, 3, 287-291.

23 L. Zhu, Q. Zhang, H. H. Feng, S. Ang, F. S. Chauc and W. T. Liu, Lab Chip, 2004, 4, 337-341.

24 A. Valero, F. Merino, F. Wolbers, R. Luttge, I. Vermes, H. Andersson and A. Van Den Berg, Lab Chip, 2005, 5, 49-55.

25 D. Di Carlo, L. Y. Wu and L. P. Lee, Lab Chip, 2006, 6, 1445-1449.

26 A. Y. Fu, H. P. Chou, C. Spence, F. H. Arnold and S. R. Quake, Anal. Chem., 2002, 74, 2451-2457.

27 A. R. Wheeler, W. R. Throndset, R. J. Whelan, A. M. Leach, R. N. Zare, Y. H. Liao, K. Farrell, I. D. Manger and A. Daridon, Anal. Chem., 2003, 75, 3581-3586.

28 A. Tourovskaia, X. Figueroa-Masot and A. Folch, Lab Chip, 2005, 5, 14-19.

29 J. Ryley and O. M. Pereira-Smith, Yeast, 2006, 23, 1065-1073.

30 S. Choi, S. Song, C. Choi and J. K. Park, Lab Chip, 2007, 7, 1532 1538.

31 M. A. Unger, H. P. Chou, T. Thorsen, A. Scherer and S. R. Quake, Science, 2000, 288, 113-116.

32 P. J. Hung, P. J. Lee, P. Sabounchi, R. Lin and L. P. Lee, Biotechnol. Bioeng., 2005, 89, 1-8.

33 S. K. Murthy, P. Sethu, G. Vunjak-Novakovic, M. Toner and M. Radisic, Biomed. Microdevices, 2006, 8, 231-237.

34 D. J. Beebe, J. S. Moore, J. M. Bauer, Q. Yu, R. H. Liu, C. Devadoss and B. H. Jo, Nature, 2000, 404, 588-590.

35 J. Moorthy and D. J. Beebe, Lab Chip, 2003, 3, 62-66.

36 N. A. Peppas, J. Z. Hilt, A. Khademhosseini and R. Langer, Adv. Mater., 2006, 18, 1345-1360.

37 A. Khademhosseini, J. Yeh, S. Jon, G. Eng, K. Y. Suh, J. A. Burdick and R. Langer, Lab Chip, 2004, 4, 425-430.

38 A. Khademhosseini, J. Yeh, G. Eng, J. Karp, H. Kaji, J. Borenstein, O. C. Farokhzad and R. Langer, Lab Chip, 2005, 5, 1380-1386.

39 J. C. Love, J. L. Ronan, G. M. Grotenbreg, A. G. Van Der Veen and H. L. Ploegh, Nat. Biotechnol., 2006, 24, 703-707.

40 M. C. Park, J. Y. Hur, K. W. Kwon, S. H. Park and K. Y. Suh, Lab Chip, 2006, 6, 988-994.

41 S. H. Lee, H. E. Jeong, M. C. Park, J. Y. Hur, H. S. Cho, S. H. Park and K. Y. Suh, Adv. Mater., 2008, 20, 788-792.

42 E. Martines, K. McGhee, C. Wilkinson and A. Curtis, IEEE Trans. NanoBiosci., 2004, 3, 90-95.

43 C. H. Choi and C. J. Kim, Phys. Rev. Lett., 2006, 96, 066001.

44 K. W. Kwon, S. S. Choi, S. H. Lee, B. Kim, S. N. Lee, M. C. Park, P. Kim, S. Y. Hwang and K. Y. Suh, Lab Chip, 2007, 7, 1461-1468.

45 M. Yamada and M. Seki, Lab Chip, 2005, 5, 1233-1239.

46 M. Yamada, K. Kano, Y. Tsuda, J. Kobayashi, M. Yamato, M. Seki and T. Okano, Biomed. Microdevices, 2007, 9, 637-645.

47 L. R. Huang, E. C. Cox, R. H. Austin and J. C. Sturm, Science, 2004, 304, 987-990.

48 J. A. Davis, D. W. Inglis, K. J. Morton, D. A. Lawrence, L. R. Huang, S. Y. Chou, J. C. Sturm and R. H. Austin, Proc. Natl. Acad. Sci. U. S. A., 2006, 103, 14779-14784.

49 N. Pamme, Lab Chip, 2007, 7, 1644-1659.

50 S. Takayama, J. C. McDonald, E. Ostuni, M. N. Liang, P. J. A. Kenis, R. F. Ismagilov and G. M. Whitesides, Proc. Natl. Acad. Sci. U. S. A., 1999, 96, 5545-5548.

51 A. Khademhosseini, K. Y. Suh, S. Jon, G. Eng, J. Yeh, G. J. Chen and R. Langer, Anal. Chem., 2004, 76, 3675-3681.

52 J. Heo, K. J. Thomas, G. H. Seong and R. M. Crooks, Anal. Chem., $2003,75,22-26$.

53 J. Yang, C. W. Li and M. S. Yang, Lab Chip, 2004, 4, 53-59.

54 J. R. Rettig and A. Folch, Anal. Chem., 2005, 77, 5628-5634.

55 M. Deutsch, A. Deutsch, O. Shirihai, I. Hurevich, E. Afrimzon, Y. Shafran and N. Zurgil, Lab Chip, 2006, 6, 995-1000. 
56 T. S. Sammarco and M. A. Burns, AlChE J., 1999, 45, 350-366.

57 D. Juncker, H. Schmid, W. H. Drechsler and B. Michel, Proc. MicroTAS 2002, Nara, Japan, November 2002 pp. 952-954.

58 Y. N. Xia, Y. D. Yin, Y. Lu and J. McLellan, Adv. Funct. Mater., 2003, 13, 907-918.

59 T. Kraus, L. Malaquin, H. Schmid, W. Riess, N. D. Spencer and H. Wolf, Nature Nanotech., 2007, 2, 570-576.

60 P. A. Kralchevsky and K. Nagayama, Langmuir, 1994, 10, 23-36.

61 A. W. Adamson and A. P. Gast, Physical Chemistry of Surfaces, Wiley, New York, 1997.

62 D. Quere, Nat. Mater., 2002, 1, 14-15.

63 B. N. J. Persson, O. Albohr, U. Tartaglino, A. I. Volokitin and E. Tosatti, J. Phys. Condens. Matter, 2005, 17, R1-R62.

64 C. Neinhuis and W. Barthlott, Ann. Bot., 1997, 79, 667-677.

65 P. Ball, Nature, 1999, 400, 507-509.

66 A. B. D. Cassie and S. Baxter, Trans. Faraday Soc., 1944, 40, 546-551.

67 B. Geiger, A. Bershadsky, R. Pankov and K. M. Yamada, Nat. Rev. Mol. Cell Biol., 2001, 2, 793-805.

68 F. J. Alenghat and D. E. Ingber, Sci. STKE, 2002, 2002, PE6.
69 C. G. Galbraith and M. P. Sheetz, Curr. Opin. Cell Biol., 1998, 10, 566-571.

70 A. M. Rajnicek, S. Britland and C. D. McCaig, J. Cell Sci., 1997, 110, 2905-2913.

71 C. Oakley and D. M. Brunette, J. Cell Sci., 1993, 106, 343-354.

72 C. S. Chen, M. Mrksich, S. Huang, G. M. Whitesides and D. E. Ingber, Science, 1997, 276, 1425-1428.

73 J. L. Tan, J. Tien, D. M. Pirone, D. S. Gray, K. Bhadriraju and C. S Chen, Proc. Natl. Acad. Sci. U. S. A., 2003, 100, 1484-1489.

74 N. Zaari, P. Rajagopalan, S. K. Kim, A. J. Engler and J. Y. Wong, Adv. Mater., 2004, 16, 2133-2137.

75 P. Kim, D. H. Kim, B. Kim, S. K. Choi, S. H. Lee, A. Khademhosseini, R. Langer and K. Y. Suh, Nanotechnology, 2005, 16, 2420-2426.

76 H. E. Jeong, S. H. Lee, J. K. Kim and K. Y. Suh, Langmuir, 2006, 22 $1640-1645$.

77 A. M. Taylor, S. W. Rhee, C. H. Tu, D. H. Cribbs, C. W. Cotman and N. L. Jeon, Langmuir, 2003, 19, 1551-1556.

78 P. J. Hung, P. J. Lee, P. Sabounchi, N. Aghdam, R. Lin and L. P. Lee, Lab Chip, 2005, 5, 44-48. 